\title{
Using Relay Nodes in Wireless Sensor Networks: A Review
}

\author{
Mustapha Reda Senouci \\ Ecole Militaire Polytechnique, \\ BP 17, 16046, Bordj El-Bahri, \\ Algiers, Algeria \\ Email: mrsenouci@gmail.com
}

\author{
Mostefa Zafer \\ Ecole nationale Supérieure d'Informatique, \\ BP 68M, 16309, Oued-Smar, \\ Alger, Algérie, \\ Email: mzzafer@esi.dz
}

\author{
Mohamed Aissani \\ Ecole Militaire Polytechnique, \\ BP 17, 16046, Bordj El-Bahri, \\ Algiers, Algeria \\ Email: maissani@gmail.com
}

\begin{abstract}
To extend the lifetime of wireless sensor networks, recent works suggest the use of relay nodes. This paper surveys and examines representative approaches dealing with relay nodes deployment. It also discusses their shortcomings and presents a comparative study. Additionally, this paper provides a set of remarks and recommendations to improve the usage of relay nodes in wireless sensor networks and highlights open issues that need further investigation.
\end{abstract}

\section{INTRODUCTION}

A WIRELESS Sensor Network (WSN) is composed of Sensor Nodes (SNs) and Collector Nodes (CNs), deployed in a well-defined geographical area, called Region of Interest (RoI), to monitor the occurrence and/or evolution of a target event [1]. Each SN is responsible for collecting data associated with this event, via its sensing unit, and communicating them, using its wireless communication interface, to one of the CNs, directly, if the latter is in its communication range, or through a multi-hop routing, with the contribution of other intermediate SNs [1]

The successful completion of the control/monitoring mission assigned to the WSN requires that the network should be deployed and managed in a rigorous manner, which guarantees the RoI coverage and the WSN connectivity throughout the assigned mission [1]. The coverage must be of a predetermined order $k(k \geq 1)$, where each point of the RoI is covered by at least $k$ SNs. Likewise, the connectivity must be of a predetermined degree $l(l \geq 1)$, which means that each SN has $l$ disjoint paths, connecting it to the CNs. Usually, a redundancy in coverage and connectivity is provided $(k, l \geq 2)$ to ensure fault-tolerance.

In the WSN, the SNs are responsible, on the one hand, for collecting data related to the target event, and on the other hand, for forwarding it to the CNs. These two energy-hungry activities limit the lifetime of SNs, and lead sometimes, to the loss of coverage and/or connectivity [1]. Moreover, SNs have limited communication range, which requires, in most cases, the use of multi-hop routing, where packets go through multiple SNs before reaching the CNs, which increases the overall delivery latency and data loss rate.

To overcome the aforementioned challenges, some recent WSNs architectures [2], [3] include, in addition to SNs and CNs, relay nodes (RNs) that benefit from extended energy autonomy and a communication range greater than that of SNs. In WSNs, these RNs are intended, in particular, to preserve [4], [5] or to restore [6], [7] the network connectivity, by actively participating in the forwarding of the collected data from the SNs to the CNs. This allows to balance the load between the SNs and the RNs [8], [9], [10], and thus prolong the network lifetime.

Nevertheless, the realistic and efficient utilization of RNs in WSNs is a long process that is in its early stage, and it currently faces several challenges [11], [12], [13]. It is a process consisting of several phases. In the first place, the appropriate usage mode of RNs should be selected. After that, the next phase chooses the topology of the network, according to the context, the constraints, and the desired objectives, before starting the RNs deployment phase. This latter is an NP-Hard problem [11], [12], [13] that consists of determining the number and the positions of RNs in the RoI. Finally, to extend the lifetime of the deployed WSN, a last phase designed to optimally manage the built topology should be considered.

In this paper, we clearly define the contours of the problem of using RNs in WSNs, by surveying and discussing representative approaches dealing with RNs deployment. This survey, intended to help researchers to quickly understand existing works, is completed by a set of remarks and recommendations to improve the usage of RNs in WSNs.

The rest of this paper is organized as follows. Section II presents the operating modes that describe the possible situations of RNs usage that can occur in practice. Then, the objectives and constraints of the problem at hand are identified in Section III. Section IV is devoted to the description of the possible WSN topologies in the presence of RNs. Section V surveys existing RNs deployment methods, and discusses their underlying assumptions. Next, Section VI summarizes the reviewed approaches, discusses their shortcomings, presents a comparative study, and highlights open issues. Finally, Section VII concludes the paper.

\section{Operating Modes}

Guaranteeing the WSN connectivity throughout the planned monitoring/control mission remains the main motivation for RNs usage, despite their relatively high cost compared to that of SNs [14], [15], because the loss of connectivity may lead 
to the failure of the mission assigned to the network. Ensuring network connectivity is achieved through two modes of RNs usage: reactive mode and proactive mode.

\section{A. Reactive Mode}

This mode of usage occurs when the WSN, consisting of SNs and CNs, is already deployed in the RoI (Fig. 1(a)). As time goes by, the battery-powered SNs will gradually be energy-exhausted and begin to disappear from the network, thereby leaving in their places what is commonly called coverage voids [1]. The number and sizes of these latter will expand gradually, causing sometimes the partitioning of the WSN into completely disjointed subnetworks (Fig. 1(b)). In this situation, some still operational SNs cannot communicate their data to any $\mathrm{CN}$. It is at this moment that RNs should be deployed at specific locations [16], [17], [18], to restore the network connectivity, ensuring that each $\mathrm{SN}$ is able again to transmit its data to at least one $\mathrm{CN}$ (Fig. 1(c)). Thus, the deployed RNs act as gateways that interconnect the pieces of the network.

\section{B. Proactive Mode}

Sometimes, the use of the RNs along with SNs and CNs is considered at the network setup [16], [17], [18]. This is a precautionary measure by which the network administrator aims to preserve the coverage and the connectivity, throughout the planned monitoring/control mission [6], [7]. In fact, the RNs participate, from the beginning, in the routing of the data from SNs to CNs. In this way, SNs are, partially or totally, unloaded from the routing activity, which allows to extend their lifetimes, and thus, preserve the coverage quality of the RoI. On the other hand, the participation of RNs in data routing has a much lesser impact on their lifetimes, compared to SNs, because RNs have extended energy, which allows preserving the WSN connectivity.

The proactive usage of RNs is better than its reactive counterpart, in the sense that it maintains both coverage and connectivity, through load balancing between SNs and RNs starting from the network setup. However, it is not meant for emergency situations, where the restoration of connectivity should be immediate.

In practice, the choice between these two RNs usage modes is dictated by the order of priority given to each targeted objective (coverage, connectivity, cost, etc.), the constraints specific to the control mission (budget, duration, urgency, etc.), the constraints imposed sometimes by the RoI (a single area or several geographically distant areas) and by the functional characteristics of the employed SNs and RNs (communication range, storage capacity, etc.). The next section identifies the objectives and constraints commonly considered in the literature.

\section{OBJectives AND CONSTRAints}

Connectivity is not the only reason behind the usage of RNs in WSNs. Some existing works not only seek to preserve simple connectivity (1-connectivity) but attempt to provide fault-tolerance, by deploying a sufficient number of RNs, so that each SN will have $l(l \geq 2)$ disjoint paths to forward its data to CNs [4], [5], [9], [10], [14], [19], [20], [21]. In this way, it would be possible to substitute, in the opportunistic moment, a broken or overloaded path, by another more appropriate path.

In addition to connectivity and fault-tolerance, the usage of RNs has other objectives such as (1) the minimization of cost, through the minimization of the number of RNs to be deployed [4], [5], [11]; (2) the minimization of the packet delivery delay [8], [13], [22], [23], [24], by ensuring that the routing paths are as short as possible; (3) the maximization of the network lifetime, notably by preserving the energy of SNs [2], [4], [11], [25] and/or by using RNs powered by green energy (where they harvest large amounts of ambient energy) [5], [16], [26], [27], and (4) the maximization of the communications links quality [22], [27], [28], which allows to avoid or to minimize the packet re-transmission operations that have a negative impact on the energy consumption and on the packets delivery delay [1].

Sometimes, some of the above-mentioned objectives are taken into account in the form of constraints. These latter could be related to the budget allocated to the mission, and are usually implemented by limiting the number of RNs to be deployed [2], [3], [17], [27]. Considered constraints could also be associated with the real-time nature of the intended application, and are carried out by requiring that the packets delivery delay is always below a tolerable threshold [8], [13], [29]. Other types of constraints are imposed by the RoI, such as limiting the possible positions of RNs [3], [4], [5], [8], [12], [19]. This last constraint often occurs in real-life applications, because the RoI can include hostile or inappropriate places, where it would be difficult, or even impossible, to place the RNs. Also, the positions to be occupied by the RNs are always dependent on the placement of SNs and CNs in the RoI, as the main role of the RNs is to restore and/or maintain the WSN connectivity.

Of course, some objectives are contradictory, such as faulttolerance and deployment cost, and some others are perfectly correlated, such as the quality of communication links and the energy consumption of SNs and RNs. The definition of an order of priority among these objectives, which takes into account the various constraints and the correct relationship between them, remains necessary for efficient usage of RNs. The next section provides an analysis of the different WSNs topologies considered in the literature to meet the desired objectives.

\section{WSN TOPOLOGIES}

The objectives, the constraints, as well as the RNs usage mode adopted to satisfy them, all must be considered when selecting, thereafter, the adequate topology of the WSN. This topology, which defines the communications among the various nodes and the role of $\mathrm{SNs}$ and $\mathrm{RNs}$ in the data routing operation, have three possible forms, namely, the 1-tier topology, the 2-tier topology, and the hybrid topology. 


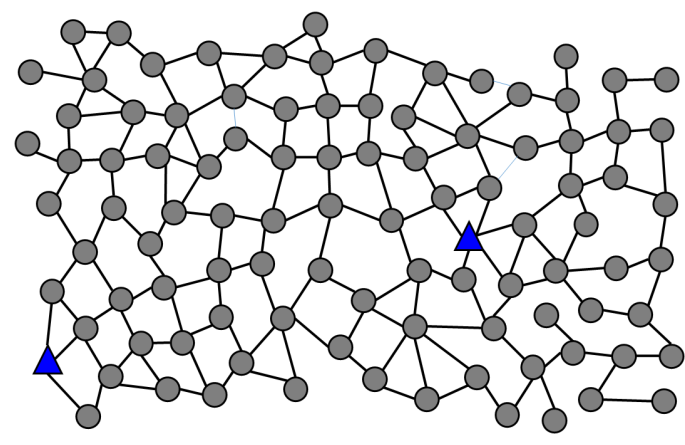

(a)

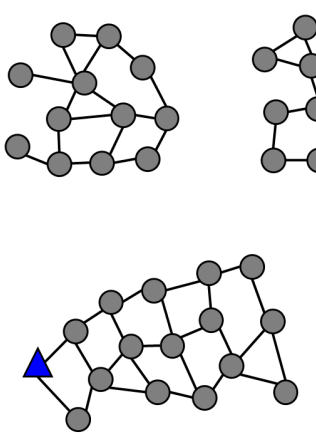

(b)

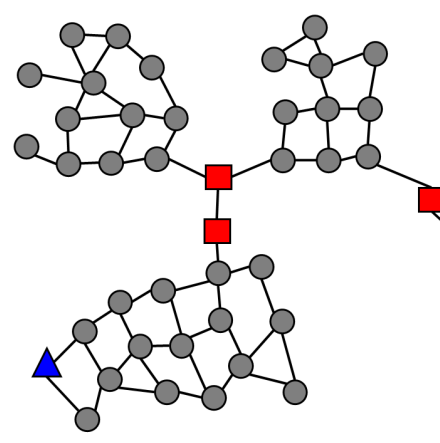

(c)

Fig. 1. Connectivity restoration by using RNs.

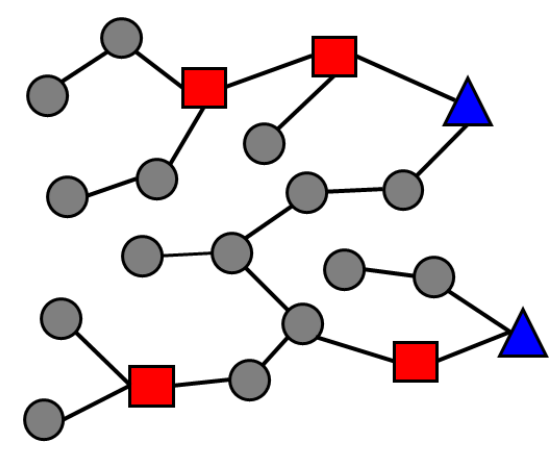

(a) 1-tier

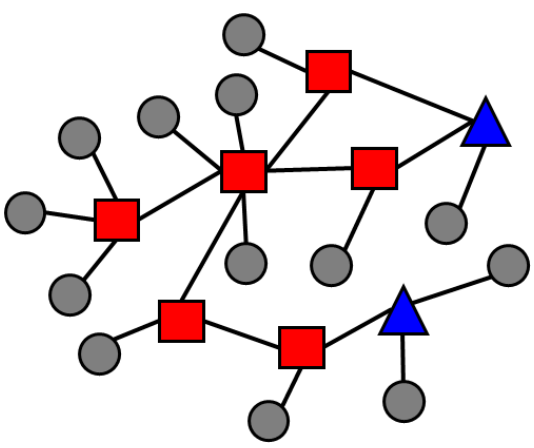

(b) 2-tier

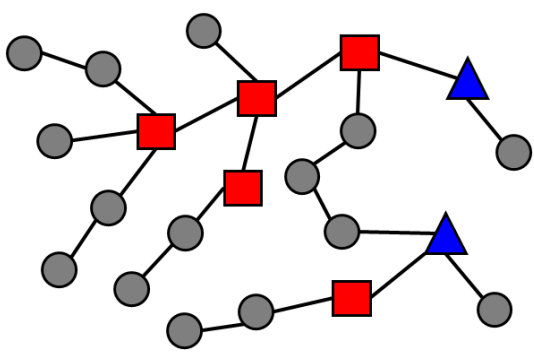

(c) Hybrid

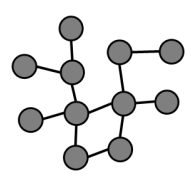

$\Delta$

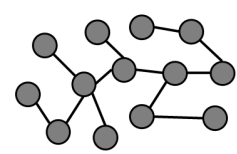

O SN

$\square$ RN

$\triangle \mathrm{CN}$

$\mathrm{OSN} \square \mathrm{RN} \quad \triangle \mathrm{CN}$

Fig. 2. WSN topologies composed of SNs, RNs, and CNs.

\section{A. 1-tier topology}

In this topology, shown in Fig. 2(a), SNs are involved, together with RNs, in the forwarding of data to CNs [6], [8], [11], [16], [17], [19], [28]. Thus, the intermediate nodes, forming the routing path connecting a $\mathrm{SN}$ to a $\mathrm{CN}$, can be $\mathrm{SNs}$ and/or RNs. Typically, this 1-tier topology is selected when a reactive usage of RNs is assumed, more specifically, when RNs are merely used to restore the WSN connectivity. In this manner, RNs potential positions are limited and are located in the voids separating the pieces of the disconnected WSN [8],
[16], [18], [21].

\section{B. 2-tier topology}

In this topology, illustrated in Fig. 2(b), each SN deals only with the collection of data and transmits it to one of the CNs or, if necessary (no $\mathrm{CN}$ is within its communication range), to one of the RNs within its communication range. Subsequently, the RNs take care of routing this data to CNs [4], [5], [12], [13], [14]. Thus, the intermediate nodes, constituting the routing path connecting a $\mathrm{SN}$ to a $\mathrm{CN}$, are necessarily RNs. 
This 2-tier topology is seen as a clustering scheme of the WSN [4], [11], [14], [24], [30], where each cluster has a $\mathrm{RN}$, acting as a cluster-head, and many SNs, acting as cluster members. This topology correlates much more with a proactive usage of RNs [5], [10], [27] because the positions to be occupied by the RNs should be well distributed over the entire RoI, so that each SN can communicate with at least one RN. In this way, it would be possible to build the necessary clusters based on RNs, such as all the cluster-heads are RNs [2], [4], [11], [14], [24], [30].

In comparison with the 1-tier topology, the 2-tier topology is more promising in terms of packets delivery delay and loss rate [8], [13], [22]. This is due to the fact that the routing paths, consisting only of RNs that have a communication range exceeding that of SNs, become shorter. Also, the 2-tier topology extends the lifespan of SNs [4], since these latter are totally unloaded from the routing task. In return, the 2-tier topology is costly, since its construction requires more RNs [4], [5], [19]. To address this gap, approaches adopting this 2tier topology endeavor to minimize the number of used RNs.

\section{Hybrid topology}

To take advantage of the aforementioned benefits of the 2-tier topology while minimizing the number of used RNs, some approaches [24], [25] adopt another topology, that we qualified it as hybrid. This hybrid topology resembles the 2tier topology, except that the communication between a $\mathrm{SN}$ and its closest $\mathrm{RN}$ is performed, if necessary (RN is not in the communication range of the $\mathrm{SN}$ ), via other intermediate SNs (see Fig. 2(c)). Thus, data collected by a SN can traverse a set of intermediate SNs, before reaching the first RN, which accomplish, together with some other RNs, the rest of the routing operation, similarly to the case when the 2-tier topology is used.

\section{RNS DEPLOYMENT APPROACHES}

It should be noted that, unlike SNs that can be deployed randomly or deterministically [1], RNs are always deployed in a deterministic way, given the main following considerations: (i) $\mathrm{RNs}$ are responsible for forwarding data from $\mathrm{SNs}$ to CNs, whose positions must be computed according to the locations already occupied by SNs and CNs [31]; (ii) RNs are significantly more expensive than SNs. Thus, in comparison to a random deployment, a deterministic deployment allows to better optimize the total number of RNs.

The deterministic deployment of RNs consists in computing the number and the appropriate positions of the RNs in the RoI, which allow to reach the sought objectives and to respect the considered constraints while taking into account the assumed topology [4], [7], [28]. This problem has been shown to be computationally NP-hard [2], [3], [11], whose resolution involves two phases: a formulation phase and a resolution phase. The formulation phase describes, on the basis of the adopted assumptions, the relation between the different constraints and objectives, whereas the resolution phase, which is based on heuristics or meta-heuristics, selects from the set of possible solutions, one solution that meets the desired objectives.

It should be noted that one of the most important assumptions that determine the soundness and practicability of a proposed deployment approach are those in relation with the adopted communication model. The latter describes, in a binary [2], [6], [11], [12], [17] or probabilistic manner [3], [16], [22], [26], [28], the quality of a communication link between two nodes, according to a set of parameters, which group in most of the proposed approaches, the distance between the communicating nodes [2], [3], [11], [12], [22], the transmission range (power) of nodes [2], [3], [11], [12], [22], the obstacles [32] as well as the medium of transmissions (radio, acoustic, etc.) [3].

In the related literature, there are different formulations of the deterministic deployment of RNs such as STP (Steiner Tree Problem) [4], [12], [17], [19], [22], CDS (Connected Dominating Set) [11], [25], SCP (Set Cover Problem) [8], [9], [13], [30], MST (Minimum Spanning Tree) [10] or ILP (Integer Linear Programming) [5], [7], [24], [26], [33]. Some other RNs deployment approaches [2], [14], [16], [28] come up with their proper formulation of the problem at hand. The formulated RNs deployment problem is solved through metaheuristics such as the GA (Genetic Algorithms) [2], [20], the GSA (Gravitational Search Algorithm) [2], [18], [27], the DE (Differential Evolution) [27], the PSO (Particle Swarm Optimization) [18], the Column Generation (CG) algorithm [33] or other existing heuristics [34], [35], [36], [37], [38]. Some others RNs deployment approach [13], [14], [16], [26], [28] devise their proper heuristics to solve the problem at hand.

It should be remembered that the effectiveness of a RNs deployment approach depends on each of the above-mentioned phases, where the practicalness of an approach is strongly linked to the considered assumptions and constraints, as well as to the formulation phase describing their influence on the intended objectives. Moreover, the degree of satisfaction of the desired objectives, once they are correctly modeled in the formulation phase, depends on the efficiency of the adopted resolution method, which denotes its capacity to explore many possible solutions and to converge, quickly, towards a goodquality solution.

\section{Synthesis AND OPEN ISSUES}

The introduction of RNs within WSNs in order to preserve or restore connectivity, in addition to other objectives, is a delicate task, which requires in the first place a clear definition of the objectives and an inventory of the various constraints, to determine the proper operating mode (reactive or proactive). Taking into account the latter, and without disregarding the constraints and the searched objectives, the appropriate topology could be selected. It is at this point that the real work of deployment begins, which consists of determining the number and positions of RNs, allowing the network to operate according to the chosen topology while respecting the objectives and constraints previously identified. This process 
is illustrated in Fig. 3, which highlights the sequence and dependence among these phases.

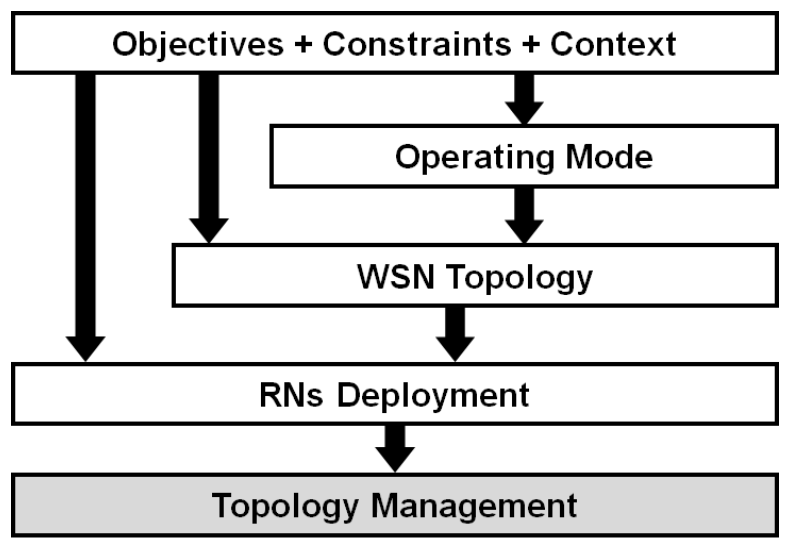

Fig. 3. Process of using RNs in WSNs.

Table I shows a comparative analysis of the most recent RNs deployment approaches. Although they start with welldefined objectives, some of these approaches have motivated the selection of the appropriate topology (1-tier, 2-tier, or hybrid) based only on the objectives. They overlooked the fact that this choice is tributary also to external constraints related to the envisaged application (budget and delay of deployment, allowed positions for RNs, urgency, etc.), which require to identify, in the first place, the appropriate operating mode (reactive or proactive) before thinking about the topology to be adopted. Due to the omission of this intermediate phase, the context of these approaches usage remains unclear.

In addition, these approaches suffer from a problem of practicalness, due to the omission of certain constraints having a quasi-permanent presence in real-life applications, or because of the adoption of unrealistic assumptions. For instance, excepting some works [3], [39], [30] that considered 3D or very specific RoI, all mentioned approaches are designed and/or evaluated under the assumption that the RoI is purely 2D, which makes them irrelevant for realistic 3D RoI, and limits their usage to very limited cases.

Furthermore, besides few works [32] that take into account, in a superficial way, the impact of obstacles, the wireless communications are modeled, in the others approaches, by very simple models that consider only the distance and the communication range of the nodes, omitting other important factors, such as obstacles, which can prevent the communications, or in the best cases, degrade their qualities. Also, some of these models are binary, forgetting the probabilistic nature of wireless communications, confirmed in several previous works.

Additionally, the constraint of RNs positions, which has a real and quasi-permanent presence, has been overlooked, or in the best cases, treated in a very superficial way, where all the allowed positions of RNs are chosen arbitrarily. However, in reality, the determination of these positions requires an analysis of the RoI. The impact of this gap will be more significant in the case of purely 3D RoI, where the topography is the first factor that imposes this kind of constraints.

Finally, a serious problem should be mentioned and it concerns the basic idea behind the use of RNs in WSNs. Indeed, the intensive participation of RNs in data routing can exhaust, in a very fast manner, their limited residual energy, especially when the 2-tier topology is considered. This aspect has not been well dealt with and remains an open issue. To handle this situation, some approaches assume that a RN has an unlimited energy resource, which remains an unrealistic assumption (at least for now). Some other approaches have aimed to ensure $k$-connectivity to face the failure of RNs, but we believe that they need to be strengthened. More precisely, we believe that the process of exploiting RNs in WSNs should be fortified by a network topology management phase (see Fig. 3), designed in particular to optimize the RNs energy consumption, by using a load balancing strategy. To the best of our knowledge, no such complete solution has been proposed. Consequently, efficient topology management of a heterogeneous WSN, composed of SNs and RNs, remains an open issue.

\section{CONCLUSION}

Recent works suggest enhancing traditional WSNs architecture, consisting of SNs and CNs, by introducing RNs to provide reliable data transport from SNs to CNs. In this paper, we have presented and discussed the steps that constitute the process of exploiting RNs in WSNs. This process starts with the definition of the desired objectives and ends with the deployment phase, which consists in determining the number and the positions of RNs in the RoI. In the end, we have carried out a comparative study among the most recent RNs deployment strategies and pointed out the main shortcomings of existing works.

The identified shortcomings are related in particular to the practicalness of these works since most of them assumed that the RoI is $2 \mathrm{D}$ and adopted unrealistic communications models, omitting the undeniable impact of some factors on wireless communications, notably obstacles. Nevertheless, the most important remark is the absence of a topology management phase that should follow the deployment of the WSN, and which helps to optimize the resources of the WSN, especially the energy of RNs that are actively involved in data routing. This phase, which must further reinforce the measures taken by some recent works that have targeted fault-tolerance (by ensuring the $k$-connectivity of the network), should notably manage the load balancing among RNs. We believe that this phase is highly important and deserves immediate attention.

\section{REFERENCES}

[1] M. R. Senouci and A. Mellouk, Deploying Wireless Sensor Networks: Theory and Practice. Elsevier, 2016.

[2] J. M. Lanza-Gutierrez and J. A. Gomez-Pulido, "A gravitational search algorithm for solving the relay node placement problem in wireless sensor networks," International Journal of Communication Systems, vol. 30, no. 2, 2015. doi: 10.1002/dac. 2957 
TABLE I

A COMPARISON BETWEEN THE MOST RECENT RNS DEPLOYMENT APPROACHES.

\begin{tabular}{|c|c|c|c|c|c|c|c|c|c|c|c|c|c|c|c|}
\hline \multirow{2}{*}{ Ref. } & \multirow{2}{*}{ RoI } & \multirow{2}{*}{$\begin{array}{l}\text { Operating } \\
\text { Mode }\end{array}$} & \multirow{2}{*}{ Topology } & \multirow{2}{*}{$\begin{array}{c}\text { Connectivity degree } \\
\qquad(k)\end{array}$} & \multicolumn{5}{|c|}{ Objectives } & \multicolumn{3}{|c|}{ Constraints } & \multicolumn{3}{|c|}{ Deployment } \\
\hline & & & & & 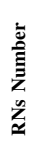 & 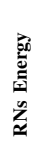 & 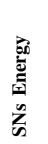 & 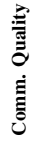 & 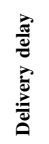 & 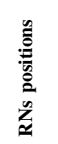 & 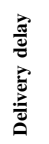 & 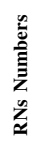 & 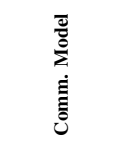 & 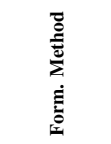 & 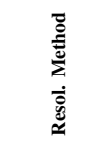 \\
\hline [2] & $2 \mathrm{D}$ & Proactive & 1-tier & $k=1$ & & & $\checkmark$ & & & & & $\checkmark$ & Binary & New & $\mathrm{GA}+\mathrm{GSA}$ \\
\hline [3] & Specific & Proactive & 2-tier & $k=1$ & $\checkmark$ & & $\checkmark$ & $\checkmark$ & & $\checkmark$ & & & Probabilistic & ILP & New \\
\hline [4] & $2 \mathrm{D}$ & Proactive & 2-tier & $k \in\{1,2\}$ & $\checkmark$ & & $\checkmark$ & & & $\checkmark$ & & & Binary & STP & [34] \\
\hline [5] & $2 \mathrm{D}$ & Proactive & 2-tier & $k \in\{1,2\}$ & $\checkmark$ & $\checkmark$ & & & & $\checkmark$ & & & Binary & ILP & [36] \\
\hline$[6],[15]$ & $2 \mathrm{D}$ & Reactive & 1-tier, 2-tier & $k=1$ & $\checkmark$ & & & & & & & & Binary & STP & {$[35]+$ New } \\
\hline [7] & $2 \mathrm{D}$ & Reactive & 1-tier & $k=1$ & & $\checkmark$ & & & & & & & Binary & ILP & New \\
\hline [9], [40] & $2 \mathrm{D}$ & Proactive & 2-tier & $k \in\{1,2\}$ & $\checkmark$ & & & & & & & & Binary & SCP & New \\
\hline [10] & $2 \mathrm{D}$ & Proactive & 2-tier & $k=2$ & & $\checkmark$ & & & & & & & Binary & MST & New \\
\hline [11], [25] & $2 \mathrm{D}$ & Proactive & 1-tier + Hybrid & $k=1$ & $\checkmark$ & & $\checkmark$ & & & & & & Binary & CDS + ILP & [36], [37] \\
\hline [12] & $2 \mathrm{D}$ & Proactive & 2-tier & $k=1$ & $\checkmark$ & & & & & $\checkmark$ & & & Binary & STP + ILP & [36] \\
\hline [14] & $2 \mathrm{D}$ & Proactive & 2-tier & $k \in\{2,3, \cdots\}$ & $\checkmark$ & & & & & & & & Binary & New & New \\
\hline$[16]$ & 2D & Reactive & 1-tier & $k=1$ & $\checkmark$ & $\checkmark$ & & & & & & & Probabilistic & New & New \\
\hline [41] & $2 \mathrm{D}$ & Proactive & 2-tier & $k=1$ & & $\checkmark$ & & $\checkmark$ & $\checkmark$ & & & & Probabilistic & New & New \\
\hline [17], [18], [42] & $2 \mathrm{D}$ & Reactive & 1-tier & $k=1$ & $\checkmark$ & & & & & & & $\checkmark$ & Binary & STP & $\mathrm{PSO}+\mathrm{New}$ \\
\hline [19] & $2 \mathrm{D}$ & Proactive & 1-tier & $k \in\{1,2\}$ & $\checkmark$ & & & & & $\checkmark$ & & & Binary & STP & [34] \\
\hline [21] & $2 \mathrm{D}$ & Proactive & 1-tier, 2-tier & $k=2$ & $\checkmark$ & & & & & & & & Binary & STP & [15] \\
\hline [22] & 2D & Proactive & 2-tier & $k=1$ & $\checkmark$ & & & $\checkmark$ & $\checkmark$ & $\checkmark$ & & & Probabilistic & STP & [34] \\
\hline [23] & $2 \mathrm{D}$ & Proactive & 2-tier & $k=1$ & $\checkmark$ & $\checkmark$ & $\checkmark$ & & & $\checkmark$ & & & Probabilistic & New & New \\
\hline [24] & $2 \mathrm{D}$ & Proactive & Hybrid & $k=1$ & $\checkmark$ & & & & $\checkmark$ & & & & Binary & ILP & New \\
\hline [26] & $2 \mathrm{D}$ & Proactive & 2-tier & $k=1$ & $\checkmark$ & $\checkmark$ & & & & $\checkmark$ & & & Probabilistic & ILP & New \\
\hline [27] & $2 \mathrm{D}$ & Proactive & 2-tier & $k=1$ & $\checkmark$ & $\checkmark$ & & $\checkmark$ & & & & $\checkmark$ & Probabilistic & ILP & $\mathrm{DE}+\mathrm{GSA}$ \\
\hline [28] & $2 \mathrm{D}$ & Proactive & 1-tier & $k=1$ & & & & $\checkmark$ & & & & $\checkmark$ & Probabilistic & New & New \\
\hline [29], [43] & $2 \mathrm{D}$ & Proactive & 1-tier & $k=1$ & $\checkmark$ & & & & $\checkmark$ & $\checkmark$ & $\checkmark$ & & Probabilistic & $\mathrm{STP}+\mathrm{New}$ & New \\
\hline [30] & Specific & Proactive & 2-tier & $k=1$ & $\checkmark$ & & & $\checkmark$ & & & & & Probabilistic & $\mathrm{SCP}$ & New \\
\hline [32] & $2 \mathrm{D}$ & Proactive & 2-tier & $k=1$ & $\checkmark$ & & & & $\checkmark$ & & & & Probabilistic & ILP & New \\
\hline [33] & $2 \mathrm{D}$ & Proactive & 1-tier & $k=1$ & $\checkmark$ & & & & $\checkmark$ & & $\checkmark$ & & Binary & ILP & $\mathrm{CG}$ \\
\hline [44] & 2D & Proactive & 1-tier & $k=1$ & & $\checkmark$ & $\checkmark$ & & $\checkmark$ & & & & Probabilistic & ILP & New \\
\hline [39] & $3 \mathrm{D}$ & Proactive & 2-tier & $k=1$ & $\checkmark$ & & & $\checkmark$ & & $\checkmark$ & & & Probabilistic & STP & [34] \\
\hline [45] & $3 \mathrm{D}$ & Proactive & 2-tier & $k \in\{2,3, \cdots\}$ & $\checkmark$ & & & $\checkmark$ & & $\checkmark$ & & & Probabilistic & STP & [34] \\
\hline [46] & $2 \mathrm{D}$ & Proactive & 2-tier & $k=1$ & & $\checkmark$ & & $\checkmark$ & $\checkmark$ & & & & Probabilistic & New & New \\
\hline
\end{tabular}


[3] D. Wu, D. Chatzigeorgiou, K. Youcef-Toumi, S. Mekid, and R. Ben-Mansour, "Channel-Aware Relay Node Placement in Wireless Sensor Networks for Pipeline Inspection," Transactions on Wireless Communications, vol. 13, no. 7, pp. 3510-3523, 2014. doi: 10.1109/TWC.2014.2314120

[4] D. Yang, S. Misra, X. Fang, G. Xue, and J. Zhang, "Two-Tiered Constrained Relay Node Placement in Wireless Sensor Networks: Computational Complexity and Efficient Approximations," Transactions on Mobile Computing, vol. 11, no. 8, pp. 1-13, 2012. doi: 10.1109/TMC.2011.126

[5] S. Misra, N. E. Majd, and H. Huang, "Approximation Algorithms for Constrained Relay Node Placement in Energy Harvesting Wireless Sensor Networks," Transactions on Computers, vol. 63, no. 12, pp. 2933-2947, 2013. doi: 10.1109/TC.2013.171

[6] H. Zeng and Z. Kang, "Relay Node Placement to Restore Connectivity in Wireless Sensor Networks," in 9th International Conference on Communication Software and Networks (ICCSN), Guangzhou, China, Dec. 2017. doi: 10.1109/ICCSN.2017.8230124 pp. 301-305.

[7] G. Xiong, L. Hong, and Y. Guangyou, "Improving Energy Efficiency by Optimizing Relay Nodes Deployment in Wireless Sensor Networks," in 9th International Conference on Communication Software and Networks (ICCSN), Guangzhou, China, Dec. 2017. doi: 10.1109/ICCSN.2017.8230125 pp. 306-310.

[8] C. Ma, W. Liang, and M. Zheng, "Set-Covering-based Algorithm for Delay Constrained Relay Node Placement in Wireless Sensor Networks," in International Conference on Communications (ICC), Kuala Lumpur, Malaysia, July 2016. doi: 10.1109/ICC.2016.7510976 pp. 1-6.

[9] C. Ma, W. Liang, M. Zheng, and H. Sharif, "A Connectivity-Aware Approximation Algorithm for Relay Node Placement in Wireless Sensor Networks," Sensors, vol. 16, no. 2, pp. 515-528, 2015. doi: 10.1109/JSEN.2015.2456931

[10] Q. Chen, Y. Hu, Z. Chen, V. Grout, D. Zhang, H. Wang, and H. Xing, "Improved Relay Node Placement Algorithm for Wireless Sensor Networks Application in Wind Farm," in International Conference on Smart Energy Grid Engineering (SEGE), Oshawa, Canada, Jan. 2013. doi: 10.1109/SEGE.2013.6707901 pp. 1-6.

[11] D. Djenouri and M. Bagaa, "Energy Harvesting Aware Relay Node Addition for Power-Efficient Coverage in Wireless Sensor Networks," in International Conference on Communications (ICC). London, UK: IEEE, Sep. 2015. doi: 10.1109/ICC.2015.7248303 pp. 86-91.

[12] A. Chelli, M. Bagaa, D. Djenouri, I. Balasingham, and T. Taleb, "One Step Approach for Two-Tiered Constrained Relay Node Placement in Wireless Sensor Networks," Wireless Communications Letters, vol. 5, no. 4, pp. 448-451, 2016. doi: 10.1109/LWC.2016.2583426

[13] C. Ma, W. Liang, and M. Zheng, "Delay Constrained Relay Node Placement in Two-tiered Wireless Sensor Networks: A Set-Coveringbased Algorithm," Journal of Network and Computer Applications, vol. 93, pp. 76-90, 2017. doi: 10.1016/j.jnca.2017.05.004

[14] K. Nitesh and P. K. Jana, "Relay Node Placement with Assured Coverage and Connectivity: A Jarvis March Approach," Wireless Personal Communications, vol. 98, no. 1, pp. 1361-1381, 2017. doi: 10.1007/s11277-017-4922-8

[15] E. L. Lloyd and G. Xue, "Relay Node Placement in Wireless Sensor Networks," Transactions on Computers, vol. 56, no. 1, pp. 134-138, 2007. doi: 10.1007/s11276-006-0724-8

[16] S. Xu, L. Jiang, C. He, and Q. Xi, "Relay Node Placement in Partitioned Wireless Sensor Networks with Guaranteed Lifetime," in Global Communications Conference (GLOBECOM), Atlanta, USA, June 2013. doi: 10.1109/GLOCOM.2013.6831078 pp. 243-248.

[17] C. Zhou, A. Mazumder, A. Das, K. Basu, N. Matin-Moghaddam, S. Mehrani, and A. Sen, "Relay Node Placement Under Budget Constraint," in 19th International Conference on Distributed Computing and Networking, Varanasi, India, Jan. 2018. doi: 10.1145/3154273.3154302 pp. 1-6.

[18] Y.-H. Xu, W.-G. Jiao, YinWu, and J. Song, "Variable-dimension swarm meta-heuristic for the optimal placement of relay nodes in wireless sensor networks," International Journal of Distributed Sensor Networks, vol. 13, no. 3, pp. 1-15, 2017. doi: 10.1177/1550147717700895

[19] S. Misra, S. D. Hong, G. L. Xue, and J. Tang, "Constrained Relay Node Placement in Wireless Sensor Networks: Formulation and Approximations," IEEE/ACM Transactions on Networking, vol. 18, no. 2, pp. 434-447, 2010. doi: 10.1109/TNET.2009.2033273

[20] M. Azharuddin and P. K. Jana, "A GA-based approach for fault tolerant relay node placement in wireless sensor networks," in Third
International Conference on Computer, Communication, Control and Information Technology (C3IT), Hooghly, India, March 2015. doi: 10.1109/C3IT.2015.7060111 pp. 1-6.

[21] W. Zhang, G. Xue, and S. Misra, "Fault-Tolerant Relay Node Placement in Wireless Sensor Networks: Problems and Algorithms," in 26th International Conference on Computer Communications, Barcelona, Spain, May 2007. doi: 10.1109/INFCOM.2007.193 pp. 1649-1657.

[22] M. Bagaa, A. Chelli, D. Djenouri, T. Taleb, I. Balasingham, and K. Kansanen, "Optimal Placement of Relay Nodes Over Limited Positions in Wireless Sensor Networks," IEEE Transactions on Wireless Communications, vol. 16, no. 4, pp. 2205-2219, 2017. doi: 10.1109/TWC.2017.2658598

[23] W. Zhu, S. Xianhe, L. Cuicui, and C. Jianhui, "Relay Node Placement Algorithm Based on Grid in Wireless Sensor Network," in Third International Conference on Instrumentation, Measurement, Computer, Communication and Control, Shenyang, China, June 2013. doi: 10.1109/IMCCC.2013.65 pp. 278-283.

[24] M. Shokrnezhad, V. Zolfaghari, and S. Khorsandi, "Relay Node Placement in Mission Critical Smart Grid Networks," in 7'th International Symposium on Telecommunications, Tehran, Iran, Sept. 2014. doi: 10.1109/ISTEL.2014.7000794 pp. 707-711.

[25] D. Djenouri and M. Bagaa, "Energy-Aware Constrained Relay Node Deployment for Sustainable Wireless Sensor Networks," Transactions on Sustainable Computing, vol. 2, no. 1, pp. 30-42, 2017. doi: 10.1109/TSUSC.2017.2666844

[26] Z. Zheng, L. X. Cai, R. Zhang, and X. S. Shen, "RNP-SA: Joint Relay Placement and Sub-Carrier Allocation in Wireless Communication Networks with Sustainable Energy," Transactions on Wireless Communications, vol. 11, no. 10, pp. 3818-3828, 2012. doi: 10.1109/TWC.2012.090312.120461

[27] B. O. Ayinde and H. A. Hashim, "Energy-Efficient Deployment of Relay Nodes in Wireless Sensor Networks Using Evolutionary Techniques," International Journal of Wireless Information Networks, vol. 25, no. 2, pp. 157-172, 2018. doi: 10.1007/s10776-018-0388-1

[28] M. Nikolov and Z. J. Haas, "Relay Placement in Wireless Networks: Minimizing Communication Cost," Transactions on Wire less Communications, vol. 15, no. 5, pp. 3587-3602, 2016. doi: 10.1109/TWC.2016.2523984

[29] A. Bhattacharya and A. Kumar, "Delay Constrained Optimal Relay Placement for Planned Wireless Sensor Networks," in 18th International Workshop on Quality of Service (IWQoS), Beijing, China, Aug. 2010. doi: 10.1109/IWQoS.2010.5542760 pp. 1-9.

[30] R. Liu, I. J. Wassell, and K. Soga, "Relay Node Placement for Wireless Sensor Networks Deployed in Tunnels," in 6th International Conference on Wireless and Mobile Computing, Networking and Communications, Niagara Falls, Canada, Nov. 2010. doi: 10.1109/WIMOB.2010.5644984 pp. 144-150.

[31] M. Zafer, M. R. Senouci, and M. Aissani, "Terrain Partitioning Based Approach for Realistic Deployment of Wireless Sensor Networks," in International Conference on Computational Intelligence and Its Applications, May 2018. doi: 10.1007/978-3-319-89743-1_37 pp. 423-435.

[32] F. M. Al-Turjman, A. E. Al-Fagih, W. M. Alsalih, and H. S. Hassanein, "A delay-tolerant Framework for Integrated RSNs in IoT," Computer Communications, vol. 36, no. 9, pp. 998-1010, 2013. doi: 10.1016/j.comcom.2012.07.001

[33] A. Nigam and Y. K. Agarwal, "Optimal Relay Node Placement in Delay Constrained Wireless Sensor Network Design," European Journal of Operational Research, vol. 233, no. 1, pp. 220-233, 2014. doi: 10.1016/j.ejor.2013.08.031

[34] L. T. Kou, G. Markowsky, and L. Berman, "A Fast Algorithm for Steiner Trees,” Acta Informatica, vol. 15, no. 2, pp. 141-145, 1981. doi: 10.1007/BF00288961

[35] Z.-X. Yang, X.-Y. Jia, J.-Y. Hao, and Y.-P. Gao, "Geometry experiment algorithm for steiner minimal tree problem," Journal of Applied Mathematics, vol. 2013, pp. 507-508, 2013. doi: 10.1155/2013/367107

[36] "Ilog cplex: Software for mathematical programming and optimization," http://www.ilog.com/products/cplex/, 2002.

[37] D. Kim, Z. Zhang, X. Li, W. Wang, W. Wu, and D.-Z. Du, “A Better Approximation Algorithm for Computing Connected Dominating Sets in Unit Ball Graphs," Transactions on Mobile Computing, vol. 9, no. 8, pp. 1108-1118, 2010. doi: 10.1109/TMC.2010.55

[38] homas H. Cormen, C. E. Leiserson, C. L. Rivest, and C. Stein, Introduction to Algorithm. MIT Press and McGraw-Hill, 2009. 
[39] M. Zafer, M. R. Senouci, and M. Aissani, "A Practical Data Driven Approach for the Deployment of WSNs on Realistic Terrains," Transactions on Emerging Telecommunications Technologies, Jan. 2019. doi: 10.1002/ett.3558

[40] C. Ma, W. Liang, M. Zheng, and H. Sharif, "A Novel Local Search Approximation Algorithm for Relay Node Placement in Wireless Sensor Networks," in Wireless Communications and Networking Conference (WCNC), New Orleans, USA, June 2015. doi 10.1109/WCNC.2015.7127693 pp. 1536-1541.

[41] F. Al-Turjman, "Optimized Hexagon-based Deployment for Large-Scale Ubiquitous Sensor Networks," Journal of Network and Systems Manage ment, vol. 26, no. 2, pp. 255-283, 2017. doi: 10.1007/s10922-017-9415-

[42] C. Zhou, A. Mazumder, A. Das, K. Basu, N. Matin-Moghaddam, S. Mehrani, and A. Sen, "Relay Node Placement Under Budget Constraint," Pervasive and Mobile Computing, vol. 53, pp. 1-12, 2019. doi: 10.1016/j.pmcj.2018.12.001

[43] A. Bhattacharya and A. Kumar, "A Shortest Path Tree Based Algorithm for Relay Placement in a Wireless Sensor Network and Its Performance Analysis," Computer Networks, vol. 71, pp. 48-62, 2014. doi 10.1016/j.comnet.2014.06.011

[44] F. Al-Turjman, "QoS-aware Data Delivery Framework for Safetyinspired Multimedia in Integrated Vehicular-IoT," Computer Communications, vol. 121, pp. 33-43, May 2018. doi 10.1016/j.comcom.2018.02.012

[45] M. Zafer, M. R. Senouci, and M. Aissani, "Fault-Tolerant Data Transport Backbone for 3D Wireless Sensor Networks," Transactions on Emerging Telecommunications Technologies, May 2019. doi: 10.1002/ett.3660

[46] F. Al-Turjman, "Cognitive Routing Protocol for Disaster-inspired Internet of Things," Future Generation Computer Systems, vol. 42, pp. 317-334, March 2017. doi: 10.1016/j.future.2017.03.014 